Tokyo J. Math.

VOL. 6, No. 2, 1983

\title{
Helical Geodesic Immersions of Compact Rank One Symmetric Spaces into Spheres
}

\author{
Kazumi TSUKADA \\ Niigata University \\ (Communicated by K. Ogiue)
}

\section{Introduction}

Let $\varphi: M \rightarrow \widetilde{M}$ be an isometric immersion of a connected complete Riemannian manifold $M$ into a Riemannian manifold $\widetilde{M}$. If, for each geodesic $\gamma$ of $M$, the curve $\varphi \circ \gamma$ in $\widetilde{M}$ has constant curvatures of osculating order $d$ which are independent of $\gamma$, then $\varphi$ is called a helical geodesic immersion of order $d$ (cf. Sakamoto [12]). In this paper we shall study a helical geodesic immersion into a unit sphere.

We give an example of a helical geodesic immersion according to Besse [2]. Suppose that the Riemannian manifold $(M, g)$ is compact. Then it has a unique kernel for the heat equation $K: M \times M \times \boldsymbol{R}_{+}^{*} \rightarrow \boldsymbol{R}$. If there exists a map $\Xi: \boldsymbol{R}_{+} \times \boldsymbol{R}_{+}^{*} \rightarrow \boldsymbol{R}$ such that $K(p, q, t)=\Xi(\delta(p, q), t)$ for every $p, q \in M$ and $t \in \boldsymbol{R}_{+}^{*}$, then $M$ is called a strongly harmonic manifold, where $\delta$ denotes the distance function. Compact symmetric spaces of rank one are its examples. Following Besse we construct a helical geodesic and minimal immersion of a strongly harmonic manifold $(M, g)$ into a unit sphere. Let $V_{k}$ be the eigenspace of the Laplacian on $(M, g)$ corresponding to the $k$-th eigenvalue $\lambda_{k}(>0)$ and $\operatorname{dim} V_{k}=m(k)+1$. We define an inner product $\langle$,$\rangle on V_{k}$ by $\langle f, h\rangle=\int_{\mu} f \cdot h d \mu, f, h \in V_{k}$. For simplicity, we normalize the canonical measure $d \mu$ of $(M, g)$ in such a way that $\int_{M} d \mu=\operatorname{dim} V_{k}$. Denote an orthonormal basis of $V_{k}$ by $\left\{f_{i}\right\}(i=0,1, \cdots, m(k))$ and define a map $\psi: M \rightarrow \boldsymbol{R}^{m(k)+1}$ by $\psi(p)=\left(f_{0}(p), \cdots, f_{m(k)}(p)\right), p \in M$. The definition of a strongly harmonic manifold implies that there exists a function $F: \boldsymbol{R}_{+} \rightarrow \boldsymbol{R}$ such that $(\psi(p), \psi(q))=\sum_{i=0}^{m(k)} f_{i}(p) f_{i}(q)=F(\delta(p, q))$. Owing to this equation, the map $\psi$ enjoys the following properties.

(i) $\psi$ is an isometric immersion of $\left(M, c_{k} \cdot g\right)$ into $R^{m(k)+1}$, where $c_{k}=\lambda_{k} / \operatorname{dim} M$.

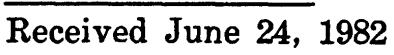


(ii) $\psi(M)$ is contained in the unit sphere $S_{1}^{m(k)}$ of $R^{m(k)+1}$ and $\psi$ is minimal in $\boldsymbol{S}_{1}^{\boldsymbol{m}(k)}$.

(iii) $\psi$ is helical geodesic in $S_{1}^{m(k)}$.

We call this map the $k$-th standard minimal immersion of a strongly harmonic manifold and denote it by $\psi_{k}$.

A helical geodesic immersion of order 1 means a totally geodesic immersion. As for order 2 and order 3, the following result is already gotten: If $\varphi: M \rightarrow S_{1}^{m}$ is a helical geodesic minimal immersion of order 2 or order 3 , then $M$ is a compact symmetric space of rank one and $\varphi$ is equivalent to a standard minimal immersion (Sakamoto [11], Nakagawa [8]). On the other hand, harmonic manifolds have been studied by many authors, but the conjecture that harmonic manifolds are rank one symmetric spaces or Euclidean spaces is still open. The study of helical geodesic immersions will be useful for the study of this conjecture. From this point of view Sakamoto ([12]) proposed the following conjecture.

ConjeCture. If $\varphi$ is a helical geodesic minimal immersion of a compact Riemannian manifold into a unit sphere, then $M$ is isometric to a compact symmetric space of rank one and $\varphi$ is equivalent to a standard minimal immersion.

In this paper we show that a helical geodesic minimal immersion of a real analytic strongly harmonic manifold into a unit sphere is equivalent to a standard minimal immersion (\$2). And we determine helical geodesic immersions of compact rank one symmetric spaces (\$3).

The author wishes to thank Professor K. Ogiue for his many valuable comments.

\section{§1. Rigidity Lemma.}

First of all we shall define the higher fundamental forms and the degree of an isometric immersion (Wallach [17]). Let $\widetilde{M}$ be a Riemannian manifold of constant curvature. Let $\varphi: M \rightarrow \widetilde{M}$ be an isometric immersion of a Riemannian manifold $M$ into $\tilde{M}$. Let $B_{2}$ be the second fundamental form of $\varphi$ at $p \in M$ and $O_{p}^{2}$ be the linear span of the image of $B_{2}$ in the normal space $N_{p}(M)$ of the immersion $\varphi$ at $p \in M$. We call $\varphi_{*} T_{p} M+O_{p}^{2}$ the second osculating space at $p \in M$. We say that $p \in M$ is degree 2 regular if $O_{p}^{2}$ is of maximal dimension. Let $R_{2} \subset M$ be the set of all degree 2 regular points of $M$. Then $R_{2}$ is open in $M$. Let $p \in R_{2}$. Let $N_{2}$ be the normal projection in $N_{p}(M)$ relative to $N_{p}(M)=$ $O_{p}^{2}+\left(O_{p}^{2}\right)^{\perp}$ (we write $\left.v \rightarrow v^{N_{2}} \in\left(O_{p}^{2}\right)^{\perp}\right)$. We define $B_{3}\left(u_{1}, u_{2}, u_{3}\right)=\left(\tilde{\nabla}_{u_{1}}\left(B_{2}\left(u_{2}, u_{3}\right)\right)\right)^{N_{2}}$ for $u_{1}, u_{2}, u_{3} \in T_{p} M$ arbitrarily extended to vector fields on $M$, where $\tilde{\nabla}$ 
denotes the Riemannian connection on $\widetilde{M} . \quad B_{3}$ is well-defined and defines a symmetric tensor field on $R_{2}$. Let $O_{p}^{3}$ be the linear span of the image of $B_{3}$. We call $B_{3}$ the third fundamental form of $\varphi$ at $p$ and $\varphi_{*} T_{p} M+$ $O_{p}^{2}+O_{p}^{3}$ the third osculating space. We call a point $p \in R_{2}$ degree 3 regular if $\operatorname{dim} O_{p}^{3}$ is maximal. We define $B_{j}, O^{j}$ for $j=2,3, \cdots$ by recursion as above on the space $R_{j-1}$ of all degree $j-1$ regular points of $M$. We call $B_{j}$ the $j$-th fundamental form of $\varphi$ and $\varphi_{*} T_{p} M+O_{p}^{2}+\cdots+O_{p}^{j}$ the $j$-th osculating space. Clearly the above process must eventually stop since $\operatorname{dim}\left(\Phi_{*} T_{p} M+O_{p}^{2}+\cdots+O_{p}^{d}\right) \leqq \operatorname{dim} T_{p} \widetilde{M}$. Let $d$ be the first integer $\geqq 2$ such that $B_{d} \equiv 0$ but $B_{d+1} \equiv 0$. Then we call $d$ the degree of $\varphi$ and the set of all $d$-regular points will be called the set of all completely regular points of $M$, denoted by $M^{\prime}=R_{d}$. In particular, when $\varphi$ is totally geodesic, i.e., $B_{2} \equiv 0$, we say that $\varphi$ has degree 1 .

In this paper, from now on we assume that the ambient space $\widetilde{M}$ is one of the standard models of Riemannian manifolds of constant curvature, namely, the Euclidean space $\boldsymbol{R}^{m}$, the sphere $S^{m}$, the real projective space $P_{m}(\boldsymbol{R})$, and the hyperbolic space $H^{m}$. It will be covenient to say that an isometric immersion $\varphi: M \rightarrow \widetilde{M}$ is full if $\varphi(M)$ is not contained in any proper totally geodesic submanifold of $\widetilde{M}$ and that two isometric immersions $\phi$ and $\hat{\phi}$ of $M$ into $\widetilde{M}$ are equivalent if there exists an isometry $\rho$ of $\tilde{M}$ such that $\rho \circ \phi=\hat{\varphi}$.

In this section we show the following rigidity lemma, which is the generalization of a Nomizu's Theorem in [9]. This result can be derived from the general treatment of connections in the bundles of normal spaces given by Kowalski [6]. But since we need the process of its proof for later applications, we shall prove it here.

LEMMA 1.1 (Rigidity Lemma). Let $\varphi$ and $\hat{\phi}$ be two full isometric immersions of a connected Riemannian manifold $M$ into $\widetilde{M}$. We assume that every point of $M$ is completely regular for both $\phi$ and $\hat{\phi}$ and that the degree of $\phi$ is equal to that of $\hat{\phi}$. If there exists a bundle isomorphism $f$ of the normal bundle $N_{\varphi}$ for $\phi$ onto the normal bundle $N_{\hat{\varphi}}$ for $\hat{\varphi}$ which preserves the bundle metrics and the higher fundamental forms, i.e., for each point $p \in M, f\left(B_{j}\left(u_{1}, \cdots, u_{j}\right)\right)=\hat{B}_{j}\left(u_{1}, \cdots, u_{j}\right), u_{1}, \cdots, u_{j} \in T_{p} M \quad 2 \leqq j \leqq$ the degree of $\phi=$ the degree of $\hat{\phi}$, where $B_{j}$ and $\hat{B}_{j}$ denote the $j$-th fundamental forms of $\phi$ and $\hat{\phi}$ respectively, then $\phi$ is equivalent to $\hat{\varphi}$.

Proof. By the assumptions of Lemma 1.1 and a Theorem of Erbacher [5], $N_{\varphi}$ and $N_{\hat{\varphi}}$ are decomposed respectively as follows:

$$
N_{\varphi}=O^{2}+\cdots+O^{d}
$$




$$
N_{\hat{\varphi}}=\hat{O}^{2}+\cdots+\hat{O}^{d},
$$

where $d=$ the degree of $\varphi=$ the degree of $\hat{\varphi}$. Since $f\left(B_{j}\left(u_{1}, \cdots, u_{j}\right)\right)=$ $\hat{B}_{j}\left(u_{1}, \cdots, u_{j}\right), f$ induces a bundle isomorphism of $O^{j}$ onto $\hat{O}^{j}$. Let $\nabla^{\perp}$ and $\hat{\nabla}^{\perp}$ be the normal connections in $N_{\varphi}$ and $N_{\hat{\varphi}}$ respectively. By Rigidity Theorem (cf. Chen [3] p. 49) it is sufficient to prove that $f$ preserves the connections. Define a linear connection $\dot{\nabla}^{\perp}$ in the normal bundle $N_{\varphi}$ as follows. For any section $\xi$ of $N_{\varphi}$ and for any vector field $X$ on $M$, we set $\dot{\nabla}_{\bar{x}}^{\frac{1}{x}} \xi=f^{-1}\left(\hat{\nabla}_{\bar{x}}^{\frac{1}{x}}(f \xi)\right)$.

Since $f$ preserves the bundle metrics, we easily see

Assertion $1 . \quad \dot{\nabla}^{\perp}$ is a metric connection of $N_{\varphi}$.

Next we show

Assertion 2. For any vector fields $X, Y$ and $Z$ on $M, \dot{\nabla}_{\bar{X}}\left(B_{2}(Y, Z)\right)=$ $\left.\nabla_{\bar{X}} \frac{1}{(} B_{2}(Y, Z)\right)$ holds.

(Proof) Using Codazzi equation, we have

$$
\begin{aligned}
\left\langle\dot{\nabla}_{\bar{X}}^{\frac{1}{X}}\right. & \left.B_{2}(Y, Z), B_{2}(U, V)\right\rangle-\left\langle\nabla \frac{1}{X} B_{2}(Y, Z), B_{2}(U, V)\right\rangle \\
& =\left\langle\hat{\nabla}_{\frac{1}{X}} \hat{B}_{2}(Y, Z), \hat{B}_{2}(U, V)\right\rangle-\left\langle\nabla^{\frac{1}{X}} B_{2}(Y, Z), B_{2}(U, V)\right\rangle \\
& =\left(\hat{\nabla}_{\frac{1}{Y}}^{1} \hat{B}_{2}(X, Z), \hat{B}_{2}(U, V)\right\rangle-\left\langle\nabla_{\frac{1}{Y}} B_{2}(X, Z), B_{2}(U, V)\right\rangle \\
& =\left\langle\dot{\nabla}_{\frac{1}{Y}} B_{2}(X, Z), B_{2}(U, V)\right\rangle-\left(\nabla_{\frac{1}{Y}} B_{2}(X, Z), B_{2}(U, V)\right\rangle,
\end{aligned}
$$

that is,

$$
\left\langle\left(\dot{\nabla}_{\bar{X}}^{\frac{1}{X}}-\nabla_{\bar{X}}^{\frac{1}{X}}\right) B_{2}(Y, Z), B_{2}(U, V)\right\rangle=\left\langle\left(\dot{\nabla}_{\bar{Y}}^{\frac{1}{Y}}-\nabla_{\frac{1}{Y}}\right) B_{2}(X, Z), B_{2}(U, V)\right\rangle .
$$

Using Assertion 1 and (1.1), we get

Therefore we have

$$
\begin{aligned}
& \left\langle\left(\dot{\nabla}_{\bar{X}}^{\frac{1}{X}}-\nabla_{\bar{X}}^{\frac{1}{X}}\right) B_{2}(Y, Z), B_{2}(U, V)\right\rangle=-\left\langle B_{2}(Y, Z),\left(\dot{\nabla}_{\bar{X}}^{\frac{1}{X}}-\nabla_{\bar{X}}\right) B_{2}(U, V)\right\rangle \\
& =-\left\langle B_{2}(Y, Z),\left(\dot{\nabla}_{U}^{\frac{1}{U}}-\nabla_{U}^{\frac{1}{U}}\right) B_{2}(X, V)\right\rangle \\
& =\left\langle\left(\dot{\nabla}_{\bar{U}}^{\frac{1}{U}}-\nabla_{U}^{\frac{1}{U}}\right) B_{2}(Y, Z), B_{2}(X, V)\right\rangle \\
& =-\left\langle B_{2}(U, Z),\left(\dot{\nabla}_{\bar{Y}}^{\frac{1}{Y}}-\nabla_{\bar{Y}}^{\frac{1}{Y}}\right) B_{2}(X, V)\right\rangle \\
& =\left\langle\left(\dot{\nabla}_{\bar{V}}^{\frac{1}{V}}-\nabla_{\bar{V}}^{\frac{1}{V}}\right) \boldsymbol{B}_{2}(U, \boldsymbol{Z}), \boldsymbol{B}_{2}(X, Y)\right\rangle \\
& =-\left\langle B_{2}(U, V),\left(\dot{\nabla}_{\frac{1}{Z}}^{\frac{1}{Z}}-\nabla_{\frac{1}{Z}}\right) B_{2}(X, Y)\right\rangle \\
& =-\left\langle B_{2}(U, V),\left(\dot{\nabla}_{\bar{X}}^{\frac{1}{X}}-\nabla_{\bar{X}}^{\frac{1}{X}}\right) B_{2}(Y, Z)\right\rangle \text {. }
\end{aligned}
$$

$$
\left\langle\dot{\nabla}_{\bar{X}}^{\frac{1}{X}} B_{2}(Y, Z), B_{2}(U, V)\right\rangle=\left\langle\nabla_{\bar{X}}^{\frac{1}{X}} B_{2}(Y, Z), B_{2}(U, V)\right\rangle .
$$

We remark that (1.1) and (1.2) are proved by using only the fact 
that there exists a bundle isomorphism $f$ of $O^{2}$ onto $\hat{O}^{2}$ which preserves bundle metrics and the second fundamental forms. Under this situation (1.1) and (1.2) show that, for a section $\xi$ of $O^{2}, f$ maps the $O^{2}$-component of $\nabla_{\bar{X}} \frac{1}{\xi}$ to the $\hat{O}^{2}$-component of $\hat{\nabla}_{\bar{X}} \frac{1}{f}(f)$. Following definitions, we get

$$
\begin{aligned}
{\left[\stackrel{\circ}{\bar{\nabla}}_{\frac{1}{X}} B_{2}(Y, Z)\right]^{N_{2}} } & =f^{-1}\left[f \dot{\nabla}^{\frac{1}{X}} B_{2}(Y, Z)\right]^{N_{2}} \\
& =f^{-1}\left[\hat{\nabla}_{\frac{1}{X}} \hat{B}_{2}(Y, Z)\right]^{N_{2}} \\
& =f^{-1} \widehat{B}_{3}(X, Y, Z) \\
& =B_{3}(X, Y, Z),
\end{aligned}
$$

that is,

$$
\left[\dot{\nabla}_{\bar{X}}^{\frac{1}{X}} B_{2}(Y, Z)\right]^{N_{2}}=B_{3}(X, Y, Z) .
$$

Since $B_{3}(X, Y, Z)=\left[\nabla_{\bar{X}}^{\frac{1}{X}} B_{2}(Y, Z)\right]^{N_{2}}$, Assertion 2 follows from (1.2) and (1.3).

Assertion 3. Generally we have $\dot{\nabla}_{\bar{X}}^{\circ} B_{j}\left(U_{1}, \cdots, U_{j}\right)=\nabla_{\bar{X}}^{\frac{1}{X}} B_{j}\left(U_{1}, \cdots, U_{j}\right)$ for the $j$-th fundamental form, $3 \leqq j \leqq d$.

(Proof) We will apply the mathematical induction. We assume that Assertion 3 holds for any $k, 2 \leqq k \leqq j-1$. Since $\dot{\nabla}^{\perp}$ is a metric connection, we get for any $k, 2 \leqq k \leqq j-1$

$$
\begin{aligned}
& \left\langle\dot{\nabla}_{\frac{1}{X}} B_{j}\left(U_{1}, \cdots, U_{j}\right), B_{k}\left(V_{1}, \cdots, V_{k}\right)\right\rangle \\
& \quad=X\left\langle B_{j}\left(U_{1}, \cdots, U_{j}\right), B_{k}\left(V_{1}, \cdots, V_{k}\right)\right\rangle-\left\langle B_{j}\left(U_{1}, \cdots, U_{j}\right), \dot{\nabla}_{\frac{1}{X}} B_{k}\left(V_{1}, \cdots, V_{k}\right)\right\rangle \\
& \quad=X\left\langle B_{j}\left(U_{1}, \cdots, U_{j}\right), B_{k}\left(V_{1}, \cdots, V_{k}\right)\right\rangle-\left\langle B_{j}\left(U_{1}, \cdots, U_{j}\right), \nabla_{\frac{1}{X}} B_{k}\left(V_{1}, \cdots, V_{k}\right)\right\rangle \\
& =\left\langle\nabla_{\frac{1}{X}} B_{j}\left(U_{1}, \cdots, U_{j}\right), B_{k}\left(V_{1}, \cdots, V_{k}\right)\right\rangle,
\end{aligned}
$$

that is,

$$
\begin{aligned}
& \left\langle\dot{\nabla}_{\frac{1}{X}} B_{j}\left(U_{1}, \cdots, U_{j}\right), B_{k}\left(V_{1}, \cdots, V_{k}\right)\right\rangle \\
& \quad=\left\langle\nabla_{\frac{1}{X}} B_{j}\left(U_{1}, \cdots, U_{j}\right), B_{k}\left(V_{1}, \cdots V_{k}\right)\right\rangle
\end{aligned}
$$

for $2 \leqq k \leqq j-1$. Since $f$ preserves metrics and the second fundamental forms, we easily see that $f^{-1} \hat{R}^{\perp}(X, Y) f \xi=R^{\perp}(X, Y) \xi$ for any section $\xi$ of $N_{\varphi}$, where $R^{\perp}$ and $\hat{R}^{\perp}$ denote the curvature tensors of $N_{\varphi}$ and $N_{\hat{\varphi}}$ with respect to $\nabla^{\perp}$ and $\hat{\nabla}^{\perp}$ respectively. We set the decomposition of $\nabla_{\bar{X}}^{\frac{1}{X}} B_{j-1}\left(U_{2}, \cdots, U_{j}\right)$ as follows:

$$
\nabla_{\bar{X}}^{\frac{1}{X}} B_{j-1}\left(U_{2}, \cdots, U_{j}\right)=B_{j}\left(X, U_{2}, \cdots, U_{j}\right)+D_{j}\left(X, U_{2}, \cdots, U_{j}\right),
$$

where $B_{j}\left(X, U_{2}, \cdots, U_{j}\right) \in O^{j}$ and $D_{j}\left(X, U_{2}, \cdots, U_{j}\right) \in O^{2}+\cdots+O^{j-1}$. By the assumption of the induction, we have 


$$
\begin{aligned}
\dot{\nabla}_{\frac{X}{X}} \dot{\nabla}_{\frac{1}{Y}} B_{j-1}\left(U_{2}, \cdots, U_{j}\right) & =\dot{\nabla}_{\frac{1}{X}}\left(\nabla_{\frac{1}{Y}} B_{j-1}\left(U_{2}, \cdots, U_{j}\right)\right) \\
& =\dot{\nabla}_{\frac{1}{X}}^{1}\left(B_{j}\left(Y, U_{2}, \cdots, U_{j}\right)+D_{j}\left(Y, U_{2}, \cdots, U_{j}\right)\right) .
\end{aligned}
$$

By the definition, we get

$$
\begin{aligned}
& \dot{\nabla}_{\bar{X}}^{\perp} \stackrel{\circ}{\nabla}_{Y}^{\perp} B_{j-1}\left(U_{2}, \cdots, U_{j}\right)-\dot{\nabla}_{\bar{Y}}^{\perp} \stackrel{\circ}{\nabla}_{\bar{X}} B_{j-1}\left(U_{2}, \cdots, U_{j}\right)-\dot{\nabla}_{[X, Y]}^{\perp} B_{j-1}\left(U_{2}, \cdots, U_{j}\right) \\
& \quad=f^{-1} \hat{R}^{\perp}(X, Y) f B_{j-1}\left(U_{2}, \cdots, U_{j}\right)
\end{aligned}
$$

and

$$
\begin{aligned}
& \nabla_{\frac{1}{X}}^{\frac{1}{Y}} \nabla_{\frac{1}{Y}} B_{j-1}\left(U_{2}, \cdots, U_{j}\right)-\nabla_{\frac{1}{Y}} \nabla_{\frac{1}{X}}^{\perp} B_{j-1}\left(U_{2}, \cdots, U_{j}\right)-\nabla_{[X, Y]}^{\perp} B_{j-1}\left(U_{2}, \cdots, U_{j}\right) \\
& \quad=R^{\perp}(X, Y) B_{j-1}\left(U_{2}, \cdots, U_{j}\right) .
\end{aligned}
$$

Noticing that $\stackrel{\circ}{\nabla}_{\bar{X}}^{\frac{1}{X}} D_{j}\left(Y, U_{2}, \cdots, U_{j}\right)=\nabla_{\bar{X}}^{\frac{1}{X}} D_{j}\left(Y, U_{2}, \cdots, U_{j}\right)$, we have

$$
\begin{aligned}
& \left\langle\dot{\nabla}_{\frac{1}{X}} B_{j}\left(Y, U_{2}, \cdots, U_{j}\right)-\dot{\nabla}_{\frac{1}{Y}} B_{j}\left(X, U_{2}, \cdots, U_{j}\right), B_{j}\left(V_{1}, \cdots, V_{j}\right)\right\rangle \\
& \quad=\left\langle\nabla_{\bar{X}} B_{j}\left(Y, U_{2}, \cdots, U_{j}\right)-\nabla_{\bar{Y}} B_{j}\left(X, U_{2}, \cdots, U_{j}\right), B_{j}\left(V_{1}, \cdots, V_{j}\right)\right\rangle,
\end{aligned}
$$

that is,

$$
\begin{aligned}
& \left\langle\left(\dot{\nabla}_{\bar{X}}^{1}-\nabla_{\left.\frac{1}{X}\right)} B_{j}\left(Y, U_{2}, \cdots, U_{j}\right), B_{j}\left(V_{1}, \cdots, V_{j}\right)\right\rangle\right. \\
& \quad=\left\langle\left(\dot{\nabla}_{\frac{1}{Y}}^{1}-\nabla_{\frac{1}{Y}}\right) B_{j}\left(X, U_{2}, \cdots, U_{j}\right), B_{j}\left(V_{1}, \cdots, V_{j}\right)\right\rangle .
\end{aligned}
$$

By the same calculations as (1.2), we have

$$
\begin{aligned}
& \left\langle\dot{\nabla}_{\frac{1}{X}} B_{j}\left(U_{1}, \cdots, U_{j}\right), B_{j}\left(V_{1}, \cdots, V_{j}\right)\right\rangle \\
& \quad=\left\langle\nabla_{\frac{1}{X}} B_{j}\left(U_{1}, \cdots, U_{j}\right), B_{j}\left(V_{1}, \cdots, V_{j}\right)\right\rangle .
\end{aligned}
$$

Again we remark that (1.4), (1.5) and (1.6) are obtained by using only the fact that there exists a bundle isomorphism $f$ of $O^{2}+\cdots+O^{j}$ onto $\hat{O}^{2}+\cdots+\hat{O}^{j}$ which preserves bundle metrics and fundamental forms. So for a section $\xi$ of $O^{2}+\cdots+O^{j}, f$ maps the $O^{2}+\cdots+O^{j}$-component of $\nabla_{\bar{x}}^{\frac{1}{x}} \xi$ to the $\hat{O}^{2}+\cdots+\hat{O}^{j}$-component of $\hat{\nabla}_{\bar{x}} f \xi$.

By the same reason as (1.3), we have

$$
\left[\dot{\nabla}_{\bar{X}}^{1} B_{j}\left(U_{1}, \cdots, U_{j}\right)\right]^{N_{j}}=B_{j+1}\left(X, U_{1}, \cdots U_{j}\right) .
$$

This, together with (1.4) and (1.6), implies that $\dot{\nabla}_{\bar{x}}^{\frac{1}{x}} B_{j}\left(U_{1}, \cdots, U_{j}\right)=$ $\nabla_{\bar{X}} B_{j}\left(U_{1}, \cdots, U_{j}\right)$ and hence Assertion 3 is proved.

By Assertion 2 and Assertion 3, $\dot{\nabla}_{\frac{1}{x} \xi} \xi \nabla_{\frac{1}{x} \xi}$ holds for any section $\xi$ of $N_{\varphi}$ and any vector field $X$ on $M$. Therefore Lemma 1.1 is proved. 
§2. Rigidity of helical geodesic minimal immersions of strongly harmonic manifolds.

As an application of Lemma 1.1, we show the following.

TheOREM 2.1. Let $\varphi$ and $\hat{\varphi}$ be two isometric immersions of a connected Riemannian manifold $M$ into a space form $\tilde{M}$. Assume that $\varphi$ is a full immersion into $\tilde{M}$ and every point of $M$ is completely regular with respect to $\varphi$. If, for any point $p \in M$ and any unit vector $x \in T_{p} M$, there exists $\varepsilon>0$ such that for the geodesic $\gamma(t)$ of $M$ which is defined on $-\varepsilon<t<\varepsilon$ and satisfies $\gamma(0)=p, \dot{\gamma}(0)=x$, the curve $\varphi \circ \gamma$ in $\tilde{M}$ is equivalent to the curve $\hat{\varphi} \circ \gamma$ in $\tilde{M}$, i.e., there exists an isometry $\rho$ of $\tilde{M}$ such that $\hat{\varphi} \circ \gamma=\rho(\varphi \circ \gamma)$, then $\varphi$ is equivalent to $\hat{\varphi}$.

REMARK. We can not remove the condition that every point of $M$ is completely regular with respect to $\varphi$. As for a counter example, we refer to Fig 10.1 (p. 366) in [14].

For the geodesic $\gamma$ of $M$ in the assumptions of the above Theorem, we set $\sigma(t)=\varphi \circ \gamma(t)$ and $\tau(t)=\hat{\varphi} \circ \gamma(t)$. Then evidently the following holds.

LEMMA 2.2. $\left\langle\widetilde{\nabla}_{\dot{\sigma}}^{(k)} \dot{\sigma}, \widetilde{\nabla}_{\dot{\sigma}}^{(l)} \dot{\sigma}\right\rangle=\left\langle\widetilde{\nabla}_{\dot{\tau}}^{(k)} \dot{\tau}, \widetilde{\nabla}_{\dot{\tau}}^{(l)} \dot{\tau}\right\rangle$, where $\widetilde{\nabla}$ denotes the Riemannian connection of $\tilde{M}$.

We denote the normal bundles for $\varphi$ and $\hat{\varphi}$ by $N_{\varphi}$ and $N_{\hat{\varphi}}$ respectively and the normal connections of $N_{\varphi}$ and $N_{\hat{\varphi}}$ by $\nabla^{\perp}$ and $\hat{\nabla}^{\perp}$ respectively. We write the covariant differentiations on $T M+N_{\varphi}$ and $T M+$ $N_{\hat{\varphi}}$ by $\bar{\nabla}$ and $\hat{\nabla}$ respectively. The assumption of the Theorem, together with Erbacher's Theorem, implies that the normal bundle $N_{\varphi}$ is decomposed as $N_{\varphi}=O^{2}+\cdots+O^{d}$, where $d$ is the degree of $\varphi$. Moreover, for $2 \leqq j \leqq d$, the $j$-th fundamental form $B_{j}$ of $\phi$ is defined on $M$. We shall prove the Theorem by calculating the higher fundamental forms $\hat{B}_{j}$ of $\hat{\varphi}$ and simultaneously constructing the bundle isomorphism $f$ of $N_{\varphi}$ onto $N_{\hat{\varphi}}$.

For an arbitrary unit tangent vector $x$ at $p$, we take the geodesic $\gamma$ of $M$ which satisfies the assumption of the Theorem. Noticing that $(\widetilde{\nabla} \dot{\sigma} \dot{\sigma})(0)=B_{2}(x, x)$ and $\left(\widetilde{\nabla}_{\dot{\tau}} \dot{\tau}\right)(0)=\hat{B}_{2}(x, x)$, we have, by Lemma $2.2,\left\langle B_{2}(x, x)\right.$, $\left.B_{2}(x, x)\right\rangle=\left\langle\hat{B}_{2}(x, x), \hat{B}_{2}(x, x)\right\rangle$ for any unit tangent vector $x$ at $p$. This equation is equivalent to

$$
\begin{aligned}
& \left\langle B_{2}\left(u_{1}, u_{2}\right), B_{2}\left(u_{3}, u_{4}\right)\right\rangle+\left\langle B_{2}\left(u_{1}, u_{3}\right), B_{2}\left(u_{4}, u_{2}\right)\right\rangle+\left\langle B_{2}\left(u_{1}, u_{4}\right), B_{2}\left(u_{2}, u_{3}\right)\right\rangle \\
& \quad=\left\langle\hat{B}_{2}\left(u_{1}, u_{2}\right), \hat{B}_{2}\left(u_{3}, u_{4}\right)\right\rangle+\left\langle\hat{B}_{2}\left(u_{1}, u_{3}\right), \hat{B}_{2}\left(u_{4}, u_{2}\right)\right\rangle+\left\langle\hat{B}_{2}\left(u_{1}, u_{4}\right), \hat{B}_{2}\left(u_{2}, u_{3}\right)\right\rangle
\end{aligned}
$$


for $u_{1}, u_{2}, u_{3}, u_{4} \in T_{p} M$.

By Gauss equation, we have

$$
\begin{aligned}
& 3\left\langle B_{2}\left(u_{1}, u_{2}\right), B_{2}\left(u_{3}, u_{4}\right)\right\rangle+\left\langle R\left(u_{1}, u_{4}\right) u_{2}, u_{3}\right\rangle \\
&+c\left\{\left\langle u_{1}, u_{2}\right\rangle\left\langle u_{4}, u_{3}\right\rangle-\left\langle u_{1}, u_{3}\right\rangle\left\langle u_{4}, u_{2}\right\rangle\right\}+\left\langle R\left(u_{1}, u_{3}\right) u_{2}, u_{4}\right\rangle \\
&+c\left\{\left\langle u_{1}, u_{2}\right\rangle\left\langle u_{3}, u_{4}\right\rangle-\left\langle u_{1}, u_{4}\right\rangle\left\langle u_{3}, u_{2}\right\rangle\right\} \\
&= 3\left\langle\hat{B}_{2}\left(u_{1}, u_{2}\right), \hat{B}_{2}\left(u_{3}, u_{4}\right)\right\rangle+\left\langle R\left(u_{1}, u_{4}\right) u_{2}, u_{3}\right\rangle \\
&+c\left\{\left\langle u_{1}, u_{2}\right\rangle\left\langle u_{4}, u_{3}\right\rangle-\left\langle u_{1}, u_{3}\right\rangle\left\langle u_{4}, u_{2}\right\rangle\right\}+\left\langle R\left(u_{1}, u_{3}\right) u_{2}, u_{4}\right\rangle \\
&+c\left\{\left\langle u_{1}, u_{2}\right\rangle\left\langle u_{3}, u_{4}\right\rangle-\left\langle u_{1}, u_{4}\right\rangle\left\langle u_{3}, u_{2}\right\rangle\right\},
\end{aligned}
$$

where $c$ is the sectional curvature of $\tilde{M}$ and $R$ denotes the curvature tensor of $M$. Thus we have

$$
\begin{aligned}
& \left\langle\hat{B}_{2}\left(u_{1}, u_{2}\right), \hat{B}_{2}\left(v_{1}, v_{2}\right)\right\rangle=\left\langle B_{2}\left(u_{1}, u_{2}\right), B_{2}\left(v_{1}, v_{2}\right)\right\rangle \\
& \text { for } u_{1}, u_{2}, v_{1}, v_{2} \in T_{p} M .
\end{aligned}
$$

We define a linear map $f$ of $O_{p}^{2}$ onto $\hat{O}_{p}^{2}$ by $f\left(B_{2}(x, y)\right)=\hat{B}_{2}(x, y), x, y \in$ $T_{p} M$. (2.1) shows that $f$ is well-defined and is an isometry of $O_{p}^{2}$ onto $\hat{O}_{p}^{2}$. Especially we have $\operatorname{dim} O_{p}^{2}=\operatorname{dim} \hat{O}_{p}^{2}$. By the assumption, every point of $M$ is degree 2 regular for $\hat{\varphi}$. Consequently $f$ is a bundle isomorphism of $O^{2}$ onto $\hat{O}^{2}$ which preserves the bundle metrics and the second fundamental forms. For convenience we denote by $D_{3}(x, y, z)\left(\operatorname{resp} . \hat{D}_{3}(x, y, z)\right)$ the $O_{p}^{2}$-component (resp. $\hat{O}_{p}^{2}$-component) of $\bar{\nabla} B_{2}(x, y, z)\left(\right.$ resp. $\hat{\nabla} \hat{B}_{2}(x, y, z)$ ). Then, by the remark in the proof of Lemma 1.1, $f\left(D_{3}(x, y, z)\right)=\hat{D}_{3}(x, y, z)$ holds for any $x, y, z$.

For the geodesic $\gamma$ of $M$ such that $\dot{\gamma}(0)=x$, we have

$$
\begin{aligned}
\widetilde{\nabla}_{\sigma}^{(2)} \dot{\sigma}(0) & =\widetilde{\nabla}_{x}\left(B_{2}(\dot{\sigma}, \dot{\sigma})\right)=\bar{\nabla} B_{2}(x, x, x)-A_{B_{2}(x, x)} x \\
& =B_{3}(x, x, x)+D_{3}(x, x, x)-A_{B_{2}(x, x)} x,
\end{aligned}
$$

where $A_{\xi}$ denotes the second fundamental tensor corresponding to the normal vector $\xi$. Then we have

$$
\left\langle\widetilde{\nabla}_{\dot{\sigma}}^{(2)} \dot{\sigma}, \widetilde{\nabla}_{\dot{\sigma}}^{(2)} \dot{\sigma}\right\rangle=\left\|B_{3}(x, x, x)\right\|^{2}+\left\|D_{3}(x, x, x)\right\|^{2}+\left\|A_{B_{2}(x, x)} x\right\|^{2} .
$$

Similarly we have

$$
\left\langle\tilde{\nabla}_{\dot{\tau}}^{(2)} \dot{\tau}, \tilde{\nabla}_{\dot{\tau}}^{(2)} \dot{\tau}\right\rangle=\left\|\hat{B}_{3}(x, x, x)\right\|^{2}+\left\|\hat{D}_{3}(x, x, x)\right\|^{2}+\left\|\hat{A}_{\hat{B}_{2}(x, x)} x\right\|^{2}
$$

By (2.1), we easily see that $A_{B_{2}(x, y)} z=\hat{A}_{\hat{B}_{2}(x, y)} z$. Since $f\left(D_{3}(x, x, x)\right)=$ $\widehat{D}_{3}(x, x, x)$, Lemma 2.2 implies that 


$$
\begin{aligned}
\left\langle\hat{B}_{3}(x, x, x), \hat{B}_{3}(x, x, x)\right\rangle= & \left\langle B_{3}(x, x, x), B_{3}(x, x, x)\right\rangle \\
& \text { for any unit tangent vector } x .
\end{aligned}
$$

Let $u, v, x_{1}, x_{2}, y_{1}$ and $y_{2}$ be tangent vectors at $p$ of $M$. We write by $\gamma$ the curve which is tangent to $u$. Let $V, X_{1}, X_{2}, Y_{1}$ and $Y_{2}$ be parallel vector fields along $\gamma$ which coincide with $v, x_{1}, x_{2}, y_{1}$ and $y_{2}$ at $p$ respectively. Then

$$
\left\langle\bar{\nabla} B_{2}\left(V, X_{1}, X_{2}\right), B_{2}\left(Y_{1}, Y_{2}\right)\right\rangle=\left\langle\hat{\nabla} \hat{B}_{2}\left(V, X_{1}, X_{2}\right), \hat{B}_{2}\left(Y_{1}, Y_{2}\right)\right\rangle
$$

holds along $\gamma$. Differentiating this equation with respect to $u$, we have

$$
\begin{aligned}
& u\left\langle\bar{\nabla} B_{2}\left(V, X_{1}, X_{2}\right), B_{2}\left(Y_{1}, Y_{2}\right)\right\rangle \\
&=\left\langle\nabla_{u}^{1}\left(\bar{\nabla} B_{2}\left(V, X_{1}, X_{2}\right)\right), B_{2}\left(Y_{1}, Y_{2}\right)\right\rangle+\left\langle\bar{\nabla} B_{2}\left(V, X_{1}, X_{2}\right), \nabla_{u}^{1}\left(B_{2}\left(Y_{1}, Y_{2}\right)\right)\right\rangle \\
&=\left\langle\bar{\nabla}^{2} B_{2}\left(u, v, x_{1}, x_{2}\right), B_{2}\left(y_{1}, y_{2}\right)\right\rangle+\left\langle\bar{\nabla} B_{2}\left(v, x_{1}, x_{2}\right), \bar{\nabla} B_{2}\left(u, y_{1}, y_{2}\right)\right\rangle \\
&=\left\langle\bar{\nabla}^{2} B_{2}\left(u, v, x_{1}, x_{2}\right), B_{2}\left(y_{1}, y_{2}\right)\right\rangle+\left\langle B_{3}\left(v, x_{1}, x_{2}\right), B_{3}\left(u, y_{1}, y_{2}\right)\right\rangle \\
&+\left\langle D_{3}\left(v, x_{1}, x_{2}\right), D_{3}\left(u, y_{1}, y_{2}\right)\right\rangle .
\end{aligned}
$$

Similarly we get

$$
\begin{aligned}
u\left\langle\hat{\nabla} \hat{B}_{2}\left(V, X_{1}, X_{2}\right), \hat{B}_{2}\left(Y_{1}, Y_{2}\right)\right\rangle & =\left\langle\hat{\nabla}^{2} \widehat{B}_{2}\left(u, v, x_{1}, x_{2}\right), \hat{B}_{2}\left(y_{1}, y_{2}\right)\right\rangle+\left\langle\hat{B}_{3}\left(v, x_{1}, x_{2}\right), \hat{B}_{3}\left(u, y_{1}, y_{2}\right)\right\rangle \\
& +\left\langle\hat{D}_{3}\left(v, x_{1}, x_{2}\right), \hat{D}_{3}\left(u, y_{1}, y_{2}\right)\right\rangle .
\end{aligned}
$$

Therefore

$$
\begin{aligned}
& \left\langle\bar{\nabla}^{2} B_{2}\left(u, v, x_{1}, x_{2}\right), B_{2}\left(y_{1}, y_{2}\right)\right\rangle+\left\langle B_{3}\left(v, x_{1}, x_{2}\right), B_{3}\left(u, y_{1}, y_{2}\right)\right\rangle \\
& \quad=\left\langle\hat{\nabla}^{2} \hat{B}_{2}\left(u, v, x_{1}, x_{2}\right), \hat{B}_{2}\left(y_{1}, y_{2}\right)\right\rangle+\left\langle\hat{B}_{3}\left(v, x_{1}, x_{2}\right), \hat{B}_{3}\left(u, y_{1}, y_{2}\right)\right\rangle
\end{aligned}
$$

holds. On the other hand, Ricci formula implies that

$$
\begin{aligned}
& \left\langle\bar{\nabla}^{2} B_{2}\left(u, v, x_{1}, x_{2}\right)-\bar{\nabla}^{2} B_{2}\left(v, u, x_{1}, x_{2}\right), B_{2}\left(y_{1}, y_{2}\right)\right\rangle \\
& \quad=\left\langle R^{\perp}(u, v) B_{2}\left(x_{1}, x_{2}\right)-B_{2}\left(R(u, v) x_{1}, x_{2}\right)-B_{2}\left(x_{1}, R(u, v) x_{2}\right), B_{2}\left(y_{1}, y_{2}\right)\right\rangle
\end{aligned}
$$

and

$$
\begin{aligned}
& \left\langle\hat{\nabla}^{2} \hat{B}_{2}\left(u, v, x_{1}, x_{2}\right)-\hat{\nabla}^{2} \hat{B}_{2}\left(v, u, x_{1}, x_{2}\right), \hat{B}_{2}\left(y_{1}, y_{2}\right)\right\rangle \\
& \quad=\left\langle\hat{R}^{\perp}(u, v) \hat{B}_{2}\left(x_{1}, x_{2}\right)-\widehat{B}_{2}\left(R(u, v) x_{1}, x_{2}\right)-\hat{B}_{2}\left(x_{1}, R(u, v) x_{2}\right), \hat{B}_{2}\left(y_{1}, y_{2}\right)\right\rangle,
\end{aligned}
$$

where $R^{\perp}$ and $\hat{R}^{\perp}$ denote the curvature tensors of $N_{\varphi}$ and $N_{\hat{\varphi}}$ respectively.

By (2.1) and Ricci equation, we get 


$$
\begin{aligned}
& \left\langle\bar{\nabla}^{2} B_{2}\left(u, v, x_{1}, x_{2}\right)-\bar{\nabla}^{2} B_{2}\left(v, u, x_{1}, x_{2}\right), B_{2}\left(y_{1}, y_{2}\right)\right\rangle \\
& \quad=\left\langle\hat{\nabla}^{2} \hat{B}_{2}\left(u, v, x_{1}, x_{2}\right)-\hat{\nabla}^{2} \hat{B}_{2}\left(v, u, x_{1}, x_{2}\right), \hat{B}_{2}\left(y_{1}, y_{2}\right)\right\rangle .
\end{aligned}
$$

Thus we have

$$
\begin{aligned}
& \left\langle\widehat{B}_{3}\left(u, x_{1}, x_{2}\right), \widehat{B}_{3}\left(v, y_{1}, y_{2}\right)\right\rangle-\left\langle\widehat{B}_{3}\left(v, x_{1}, x_{2}\right), \widehat{B}_{3}\left(u, y_{1}, y_{2}\right)\right\rangle \\
& =\left\langle B_{3}\left(u, x_{1}, x_{2}\right), B_{3}\left(v, y_{1}, y_{2}\right)\right\rangle-\left\langle B_{3}\left(v, x_{1}, x_{2}\right\rangle, B_{3}\left(u, y_{1}, y_{2}\right)\right\rangle \\
& \quad \text { for } u, v, x_{1}, x_{2}, y_{1}, y_{2} \in T_{p} M .
\end{aligned}
$$

Symmetrizing (2.2), we have

$$
\mathscr{S}_{8}\left\langle\hat{B}_{3}\left(u_{1}, u_{2}, u_{3}\right), \hat{B}_{3}\left(u_{4}, u_{5}, u_{6}\right)\right\rangle=\mathscr{S}_{6}\left\langle B_{3}\left(u_{1}, u_{2}, u_{3}\right\rangle, B_{3}\left(u_{4}, u_{5}, u_{6}\right)\right\rangle,
$$

where $\mathscr{S}_{B}$ denotes the symmetrizer of order 6 . This, together with (2.3), gives

$$
\begin{aligned}
\left\langle\hat{B}_{3}\left(u_{1}, u_{2}, u_{3}\right), \hat{B}_{3}\left(v_{1}, v_{2}, v_{3}\right)\right\rangle= & \left\langle B_{3}\left(u_{1}, u_{2}, u_{3}\right), B_{3}\left(v_{1}, v_{2}, v_{3}\right)\right\rangle \\
& \text { for } u_{1}, u_{2}, u_{3}, v_{1}, v_{2}, v_{3} \in T_{p} M .
\end{aligned}
$$

By (2.4), we can define an isometric linear map $f$ of $O_{p}^{3}$ onto $\hat{O}_{p}^{s}$ which satisfies $f\left(B_{3}(x, y, z)\right)=\widehat{B}_{3}(x, y, z)$. Especially we have $\operatorname{dim} O_{p}^{3}=$ $\operatorname{dim} \hat{O}_{p}^{3}$, which implies that every point of $M$ is degree 3 regular for $\hat{\phi}$. Consequently $f$ is a bundle isomorphism of $O^{2}+O^{3}$ onto $\hat{O}^{2}+\hat{O}^{3}$ which preserves the bundle metrics and the second and third fundamental forms.

Calculating inductively, we see that every point of $M$ is completely regular for $\hat{\phi}$ and the degree of $\hat{\phi}$ is equal to that of $\phi$. Moreover we can construct the bundle isomorphism $f$ of $N_{\varphi}$ onto $N_{\hat{\varphi}}$ which preserves the bundle metrics and the higher fundamental forms. Therefore Lemma 1.1 gives Theorem 2.1.

COROLLARY 2.3. Let $(M, g)$ be an $n$-dimensional real analytic strongly harmonic manifold. If $\phi$ is a full helical geodesic minimal immersion of $\left(M, c_{k} \cdot g\right)$ into a unit sphere $S_{1}^{l}$, then $\phi$ is equivalent to the standard minimal immersion $\psi_{k}$. In particular $l$ is equal to $m(k)$. (For notations we refer to Introduction.)

We prepare some lemmas before the proof of Corollary 2.3. Let $X \in T_{x_{0}} M-\{0\}$ and $\gamma: s \rightarrow \exp _{x_{0}}(s /\|X\|) X$ be the geodesic. Let $\left\{Y_{i}\right\} i=$ $2, \cdots, n$ be Jacobi fields along $\gamma$ such that $Y_{i}(0)=0$ for every $i$ and $\left\{Y_{i}^{\prime}(0)\right\}_{i=2, \cdots, n}$ forms an orthonormal basis of the orthogonal complement of $X$ in $T_{x_{0}} M$. Then we define $\theta: T M \rightarrow R$ by 


$$
\left\{\begin{array}{l}
\theta(0)=1 \\
\theta(X)=\|X\|^{-n+1} \operatorname{det}\left(Y_{2}(\|X\|), \cdots, Y_{n}(\|X\|)\right),
\end{array}\right.
$$

where the determinant should be understood with respect to the parallel frame field of $\left\{Y_{i}^{\prime}(0)\right\}$. It is known that a strongly harmonic manifold is a globally harmonic manifold, i.e., there exists a $C^{\infty}$-function $\Theta: \boldsymbol{R}_{+} \rightarrow$ $R$ such that $\theta(X)=\Theta(\|X\|)$ for every $x \in M$ and every $X \in T_{x} M$.

Lemma 2.4 (Berger-Gauduchon-Mazet [1] p. 134). Let $f$ be $a C^{\infty}$ function on $M$ of the form $f(x)=F\left(\delta\left(x, x_{0}\right)\right.$ ) (i.e., which depends only on the distance to $x_{0}$ ), where $\delta$ denotes the distance function. Then we have

$$
\Delta f=-\frac{d^{2} F}{d s^{2}}-\left(\frac{\theta_{x_{0}}^{\prime}}{\theta_{x_{0}}}+\frac{n-1}{s}\right) \frac{d F}{d s},
$$

where $\Delta$ denotes the Laplacian and $\theta_{x_{0}}^{\prime}$ is the radial derivative of $\theta_{x_{0}}$ in $T_{x_{0}} M$.

LeMMA 2.5 (Sakamoto [12]). Let $\varphi: M \rightarrow S_{1}^{l}$ be a helical geodesic immersion. Then there exists a $C^{\infty}$-function $F: \boldsymbol{R}_{+} \rightarrow \boldsymbol{R}$ such that the Euclidean inner product of position vectors $\varphi(x)$ and $\varphi(y)$ is given by $\langle\varphi(x), \varphi(y)\rangle=F(\delta(x, y))$.

Lemma 2.6 (Besse [2] p. 177, p. 178). Let $\varphi: M \rightarrow S_{1}^{l}$ be a helical geodesic immersion and let $\gamma: I \rightarrow M$ be a geodesic parametrized by the arc-length. Then the curvatures of the curve $\sigma=\varnothing \circ \gamma$ in $S_{1}^{l}$ are completely determined by $F^{(k)}(0), k=1,2, \cdots$, where $F$ is the function introduced in Lemma 2.5 .

Lemma 2.7 (Do Carmo and Wallach [4]). Let $\varphi:\left(M, c_{k} \cdot g\right) \rightarrow S_{1}^{l}$ be a minimal immersion. Then there exists a symmetric positive semi-definite linear map $A$ of $\boldsymbol{R}^{m(k)+1}$ such that $\phi$ is equivalent to $A \circ \psi_{k}$. Futhermore $\phi$ is equivalent to $\psi_{k}$ if and only if the associated symmetric linear mapping $A$ of $\phi$ is equal to the identity map.

Proof of Corollary 2.3. Since $l$ is not greater than $m(k)$, we may regard $\varphi$ as a helical geodesic minimal immersion into $S_{1}^{m(k)}$. We denote by $F$ and $\hat{F}$ the functions introduced in Lemma 2.5 associated with helical geodesic immersions $\psi_{k}$ and $\varphi$ respectively. For a fixed point $x_{0} \in M$, we define the functions $f$ and $\hat{f}$ on $M$ by

$$
\begin{aligned}
& f(x)=\left\langle\psi_{k}(x), \psi_{k}\left(x_{0}\right)\right\rangle=F\left(\delta\left(x, x_{0}\right)\right) \\
& \hat{f}(x)=\left\langle\varphi(x), \varphi\left(x_{0}\right)\right\rangle=\hat{F}\left(\delta\left(x, x_{0}\right)\right),
\end{aligned}
$$


where $\langle$,$\rangle denotes the Euclidean inner product of \boldsymbol{R}^{m(k)+1}$. By a Theorem of Takahashi [15], $f(x)$ and $\hat{f}(x)$ are eigenfuctions of the Laplacian on $\left(M, c_{k} \cdot g\right)$ with eigenvalue $n$. By Lemma 2.4 , we have

$$
-\frac{d^{2} F}{d s^{2}}-\frac{d F}{d s}\left(\frac{\theta_{x_{0}}^{\prime}}{\theta_{x_{0}}}+\frac{n-1}{s}\right)=n F
$$

and

$$
-\frac{d^{2} \hat{F}}{d s^{2}}-\frac{d \hat{F}}{d s}\left(\frac{\theta_{x_{0}}^{\prime}}{\theta_{x_{0}}}+\frac{n-1}{s}\right)=n \hat{F} .
$$

Since $F(0)=\hat{F}(0)=1$ and $F^{\prime}(0)=\hat{F}^{\prime}(0)=0$, we obtain $F \equiv \hat{F}$. Then Lemma 2.6 implies that, for any geodesic $\gamma$ of $M, \varnothing \circ \gamma$ is equivalent to $\psi_{k} \circ \gamma$ in $S_{1}^{m(k)}$. Let $A$ be a symmetric positive semi-definite linear map of $R^{m(k)+1}$ such that $A \circ \psi_{k}$ is equivalent to $\varphi$. Let $M^{\prime}$ be a connected open submanifold of $M$ whose points are all completely regular for $\psi_{k}$. Since $\psi_{k}$ is analytic and is a full immersion into $S_{1}^{m(k)}, \psi_{k}$ is a full immersion into $S_{1}^{m(k)}$ on $M^{\prime}$, too. We apply Theorem 2.1 to the immersions $\psi_{k}$ and $\varphi$ of $M^{\prime}$ into $S_{1}^{m(k)}$. By Lemma 2.7, $A$ is the identity map. Therefore Corollary 2.3 holds.

Since a compact symmetric space of rank one is a real analytic strongly harmonic manifold, we immediately get

COROLlary 2.8. A helical geodesic minimal immersion of a compact symmetric space of rank one into a unit sphere is equivalent to a standard minimal immersion.

This result in the case of a sphere has already been shown in [16]. space.

§3. Helical geodesic immersions of a compact rank one symmetric

First we prepare two notions.

Definition 3.1 (0'Neill [10]). Let $B$ be an $R^{k}$-valued symmetric multilinear form on $R^{n}$. We say that $B$ is isotropic if $\|B(u, \cdots, u)\|=$ constant for any unit vector $u$ in $R^{n}$.

Definition 3.2. Let $\varphi: M \rightarrow \widetilde{M}$ be an isometric immersion of a Riemannian homogeneous space $M=G / K$ into a space form $\widetilde{M}$. We say that $\phi$ is equivariant if there exists a continous homomorphism $\rho$ of $G$ into the isometry group $I(\widetilde{M})$ of $\widetilde{M}$ such that $\varphi(g \cdot p)=\rho(g) \varphi(p) p \in M, g \in G$. 
It is easily seen that the standard minimal immersion of a compact rank one symmetric space into a sphere is naturally equivariant. For the higher fundamental forms of an equivariant immersion, the following properties hold.

Lemma 3.3 (cf. Mashimo [7]). Let $\varphi: M \rightarrow \widetilde{M}$ be an equivariant isometric immersion of a Riemannian homogeneous space $M=G / K$ into a space form $\tilde{M}$. Then every point of $M$ is completely regular. Moreover the $j$-th fundamental form $B_{j}$ is G-invariant, i.e.,

$$
\begin{aligned}
B_{j \mid g p}\left(g \cdot u_{1}, \cdots, g \cdot u_{j}\right) & =\rho(g) B_{j \mid p}\left(u_{1}, \cdots, u_{j}\right) \\
\rho(g) O_{p}^{j} & =O_{g p}^{j} \\
N_{j} \circ \rho(g) & =\rho(g) \cdot N_{j}, \quad g \in G .
\end{aligned}
$$

THEOREM 3.4. Let $M$ be a compact symmetric space of rank one and $(G, K)$ be its symmetric pair. Let $\varphi: M \rightarrow S_{1}^{l}$ be an isometric immersion. Then the following three statements are mutually equivalent.

(1) $\phi$ is a G-equivariant immersion.

(2) For $2 \leqq j \leqq$ the degree of $\varphi$, the $j$-th fundamental form $B_{j}$ is constant isotropic at every degree $j-1$ regular point $p \in R_{j-1}$, i.e., there exists a positive constant $\lambda_{j}>0$ such that $\left\|B_{j}(u, \cdots, u)\right\|=\lambda_{j}$ for any unit tangent vector $u$ at $p \in R_{j-1}$.

(3) $\varphi$ is a helical geodesic immersion.

Proof. "(1) $\Rightarrow(2)$ " By Lemma 3.3, we have at $e K,\left\|B_{j}(k \cdot u, \cdots, k \cdot u)\right\|=$ $\left\|\rho(k) B_{j}(u, \cdots, u)\right\|=\left\|B_{j}(u, \cdots, u)\right\|$ for $k \in K$. Since $K$ acts transitively on the unit sphere of $T_{e K} M, B_{j}$ is isotropic at $e K$. Again by Lemma 3.3, we see that $B_{j}$ is constant isotropic.

"(1) $\Rightarrow(3) "$ For an arbitrary unit tangent vector $x$ at the origin $o=e K$ of $M$, we denote by $\gamma$ the geodesic of $M$ such that $\gamma(0)=o \dot{\gamma}(0)=$ $x$. Let $\mathfrak{g}=\mathfrak{l}+\mathfrak{m}$ be the canonical decomposition of the symmetric pair $(G, K)$. Then the geodesic $\gamma$ is described as $\gamma(t)=(\exp t X) \cdot o$, where $\exp t X$ denotes a one-parameter subgroup of $G$ and $X$ is a vector in $m$ corresponding to $x$. Since $(\varphi \circ \gamma)(t)=\rho(\exp t X) \cdot \varphi(o)$, the curve $\varphi \circ \gamma$ in $\boldsymbol{S}_{\mathbf{1}}^{\mathfrak{l}}$ has constant curvatures. Any geodesic $\gamma$ parametrized by arc-length is described as $\gamma(t)=(g \exp t X) \cdot o$ for some $g \in G$. Therefore we have $(\varphi \circ \gamma)(t)=\rho(g) \rho(\exp t X) \cdot \varphi(o)$, which gives the statement of (3).

"( 2$) \Rightarrow(1)$ " We assume without loss of generality that $\varphi$ is full. We shall prove the following.

Assertion. Under the condition of (2), we see that every point of $M$ is completely regular. That is, for $2 \leqq j \leqq$ the degree of $\varphi$, the $j$-th 
fundamental form $B_{j}$ is defined on $M$. Moreover, for any $g \in G$, we have

$$
\begin{aligned}
& \left\langle B_{j \mid g p}\left(g \cdot u_{1}, \cdots, g \cdot u_{j}\right), B_{j \mid g p}\left(g \cdot v_{1}, \cdots, g \cdot v_{j}\right)\right\rangle \\
& \quad=\left\langle B_{j \mid p}\left(u_{1}, \cdots, u_{j}\right), B_{j \mid p}\left(v_{1}, \cdots, v_{j}\right)\right\rangle, u_{1}, \cdots, u_{j}, v_{1}, \cdots, v_{j} \in T_{p} M .
\end{aligned}
$$

This Assertion is proved essentially by the same method as Theorem 2.1. For an arbitrary $g \in G$, we define another isometric immersion $\hat{\varphi}$ by $\hat{\phi}=\varphi \circ g$. Then we have

$$
\hat{B}_{j \mid p}\left(u_{1}, \cdots, u_{j}\right)=B_{j \mid g p}\left(g \cdot u_{1}, \cdots, g \cdot u_{j}\right)
$$

at a degree $j-1$ regular point $g p$ for $\varphi$, where $\hat{B}_{j}$ denotes the $j$-th fundamental form of $\hat{\phi}$. By the assumption,

$$
\left\langle B_{2}(u, u), B_{2}(u, u)\right\rangle=\left\langle B_{2}(g \cdot u, g \cdot u), B_{2}(g \cdot u, g \cdot u)\right\rangle=\left\langle\hat{B}_{2}(u, u), \hat{B}_{2}(u, u)\right\rangle
$$

holds for any unit tangent vector $u$. By the same argument as in the proof of Theorem 2.1, we obtain

$$
\left\langle\widehat{B}_{2}\left(u_{1}, u_{2}\right), \hat{B}_{2}\left(v_{1}, v_{2}\right)\right\rangle=\left\langle B_{2}\left(u_{1}, u_{2}\right), B_{2}\left(v_{1}, v_{2}\right)\right\rangle .
$$

Therefore we have

$$
\left\langle B_{2 \mid p}\left(u_{1}, u_{2}\right), B_{2 \mid p}\left(v_{1}, v_{2}\right)\right\rangle=\left\langle B_{2 \mid g p}\left(g \cdot u_{1}, g \cdot u_{2}\right), B_{2 \mid g p}\left(g \cdot v_{1}, g \cdot v_{2}\right)\right\rangle,
$$

which implies $\operatorname{dim} O_{p}^{2}=\operatorname{dim} O_{g p}^{2}$. Since $G$ acts transitively on $M$, the set of all degree 2 regular points for $\varphi$ coincides with $M$. Following the same way as the proof of Theorem 2.1, we obtain Assertion.

In the process of the proof we can construct the bundle isomorphism $f(g)$ of the normal bundle $N_{\varphi}$ for any $g \in G$ which preserves the bundle metrics and satisfies $B_{j \mid g p}\left(g \cdot u_{1}, \cdots, g \cdot u_{j}\right)=f(g) B_{j \mid p}\left(u_{1}, \cdots, u_{j}\right)$. By Lemma 1.1 , there exists an isometry $\rho(g)$ of $S_{1}^{l}$ such that $\rho(g) \circ \varphi=\varphi \circ g$. We immediately see that the map $\rho: G \rightarrow I\left(S_{1}^{l}\right)$ is a continuous homomorphism. Therefore the statement of (1) is proved.

"( 3$) \Rightarrow(2)$ " By the assumption, for any geodesic $\gamma$ of $M$ and any $g \in G$, the curve $\varphi(g \circ \gamma)$ is equivalent to the curve $\varphi(\gamma)$ in $S_{1}^{l}$. We apply the methods of Theorem 2.1 to isometric immersions $\varphi$ and $\varphi \circ g$. Moreover noticing that for two unit tangent vectors $u \in T_{p} M$ and $v \in T_{q} M$ there exists an element $g \in G$ such that $g \cdot u=v$, we obtain our result.

We construct a G-equivariant immersion of a compact rank one symmetric space $M$ into a unit sphere. Let $\psi_{k}$ be the $k$-th standard 
minimal immersion of $M$ into a unit sphere. We define

$$
\begin{aligned}
& \psi: M \rightarrow R^{m\left(k_{1}\right)+\cdots+m\left(k_{r}\right)+r} \quad \text { by } \\
& \psi(p)=\left(c_{1} \psi_{k_{1}}(p), \cdots, c_{r} \psi_{k_{r}}(p)\right) \quad p \in M,
\end{aligned}
$$

where each $c_{i}$ is a positive number and $c_{1}^{2}+\cdots+c_{r}^{2}=1$. By a suitable homothetic change of the metric of $M, \psi$ becomes an isometric immersion into a unit sphere $S_{1}^{m\left(k_{1}\right)+\cdots+m\left(k_{r}\right)+r-1} . \quad \psi$ is evidently a G-equivariant immersion. For a compact rank one symmetric space $G$ acts irreducibly on each eigenspace of the Laplacian. Therefore any $G$-equivariant isometric immersion of $M$ into a unit sphere is described as above. For convenience, we denote by $\psi_{k_{1}, \cdots, k_{r}}$ the map which is obtained as above. Here we remark that $\psi_{k_{1}, \cdots, k_{r}}$ is full in a sphere only if $k_{1}, \cdots, k_{r}$ are distinct.

By Theorem 3.4, we have

COROLlary 3.5. Let $\varphi: M \rightarrow S_{1}^{l}$ be a helical geodesic isometric immersion of a compact rank one symmetric space into a unit sphere. Assume that $\varnothing$ is full. Then there exist non-negative integers $k_{1}, \cdots, k_{r}$ such that $\varphi$ is equivalent to $\psi_{k_{1}, \cdots, k_{r}}$, where $k_{1}, \cdots, k_{r}$ are distinct and may contain zero.

\section{§4. Osculating order of a helical geodesic immersion $\psi_{k_{1}, \cdots, k_{r}}$.}

In this section we calculate the osculating order of a helical geodesic immersion $\psi_{k_{1}, \cdots, k_{r}}$ of $M=$ a sphere $S^{n}$, a complex projective space $P_{n}(C)$, or a quaternion projective space $P_{n}(\boldsymbol{H})$ into a unit sphere. We assume that $n \geqq 2$ in all case.

TheOREM 4.1. When $M=S^{n}$, the order of $\psi_{k_{1}, \cdots, k_{r}}$

$$
\text { is }\left\{\begin{array}{l}
\text { the maximum of } k_{1}, \cdots, k_{r} \text { if } k_{1}, \cdots, k_{r} \text { are all odd or all even, } \\
1+\text { the maximum of odd numbers in } k_{1}, \cdots, k_{r}+\text { the maximum of } \\
\text { even numbers in } k_{1}, \cdots, k_{r} \text { if } k_{1}, \cdots, k_{r} \text { contain both odd numbers } \\
\text { and even numbers. }
\end{array}\right.
$$

When $M=P_{n}(C)$ or $P_{n}(H)$, the order of $\psi_{k_{1}, \cdots, k_{r}}$ is $2 \times($ the maximum of $\left.k_{1}, \cdots, k_{r}\right)$.

EXAMPLES. We describe the degrees and the orders of some examples of $\psi_{k_{1}, \cdots, k_{r}}$ in the case of $S^{n}$. 


$\begin{array}{lcc} & \text { degree } & \text { order } \\ \psi_{0,1} & 2 & 2 \\ \psi_{0,2} & 2 & 2 \\ \psi_{0,8} & 3 & 4 \\ \psi_{1,2} & 3 & 4 \\ \psi_{1,3} & 3 & 3 \\ \psi_{2,3} & 4 & 6 \\ \psi_{1,2,8} & 5 & 6\end{array}$

REMARK. It is known that the degree is not greater than the order for a helical geodesic immersion (Sakamoto [12]). In the above examples there exists a helical geodesic immersion whose degree is less than the order. On the other hand, when $\psi$ is a helical geodesic minimal immersion of $M=S^{n}, P_{n}(C)$ or $P_{n}(H)$, the order is equal to the degree.

Before the proof of Theorem 4.1, we describe the eigenfunctions of the Laplacian on $S^{n}, P_{n}(C)$ and $P_{n}(H)$. Let $R^{n+1}$ be an $(n+1)$-dimensional Euclidean space $(n \geqq 1)$ with coordinates $\left(x_{0}, x_{1}, \cdots, x_{n}\right)$. Let $S^{k}\left(R^{n+1}\right)$ be the space of homogeneous polynomials of degree $k$ on $R^{n+1}$ and $H^{k}\left(R^{n+1}\right)$ be the space of harmonic homogeneous polynomials of degree $k$ on $R^{n+1}$.

LemmA 4.2 (Berger-Gauduchon-Mazet [1] p. 160). For any $k \geqq 0$, $S^{k}\left(\boldsymbol{R}^{n+1}\right)$ has the following direct decomposition:

$$
S^{2 k}\left(\boldsymbol{R}^{n+1}\right)=H^{2 k}\left(\boldsymbol{R}^{n+1}\right)+r^{2} H^{2(k-1)}\left(R^{n+1}\right)+\cdots+r^{2 k} H^{0}\left(R^{n+1}\right),
$$

and

$$
S^{2 k+1}\left(R^{n+1}\right)=H^{2 k+1}\left(R^{n+1}\right)+r^{2} H^{2 k-1}\left(R^{n+1}\right)+\cdots+r^{2 k} H^{1}\left(R^{n+1}\right),
$$

where $r^{2}=x_{0}^{2}+x_{1}^{2}+\cdots+x_{n}^{2}$.

LEMmA 4.3 (Berger-Gauduchon-Mazet [1] p. 160). The k-th eigenspace of the Laplacian on $S_{1}^{n}$ is given by the restriction of $H^{k}\left(R^{n+1}\right)$ on $S_{1}^{n}$.

Let $C^{n+1}$ be an $(n+1)$-dimensional complex Euclidean space with coordinates $\left(z_{0}, z_{1}, \cdots, z_{n}\right)$ and $H^{k, k}$ be the space of harmonic homogeneous polynomials of type $(k, k)$. Let $\pi: S^{2 n+1} \rightarrow P_{n}(C)$ be the Hopf fibration. It is known that the space given by the restriction of $H^{k, k}$ on $S^{2 n+1}$ is $\pi$ invariant ([1] p. 172). Then it is regarded as the space of functions on $P_{n}(C)$, which is denoted by $\hat{H}^{k, k}$. Let $\boldsymbol{H}^{n+1}$ be an $(n+1)$-dimensional quaternion Euclidean space and $\boldsymbol{H}^{n+1}$ be naturally regarded as $2(n+1)$ - 
dimensional complex Euclidean space $C^{2(n+1)}$. This identification gives the Riemannian submersion $\pi: P_{2 n+1}(C) \rightarrow P_{n}(H)$ with totally geodesic fibres $P_{1}(C)=S^{2}$.

Lemma 4.4 ([1] p. 173, Smith [13]). The k-th eigenspace of the Laplacian on $P_{n}(C)$ is given by $\hat{H}^{k, k}$. There exists the subspace of $\hat{H}^{k, k}$ which is invariant by the fibration $\pi: P_{2 n+1}(C) \rightarrow P_{n}(H)$ and it gives the $k$-th eigenspace of the Laplacian on $P_{n}(H)$.

REMARK. Let $H_{R}^{k, k}$ be the subspace of $H^{k, k}$ which consists of realvalued functions. Then we have $H_{R}^{k, k} \bigotimes_{R} C=H^{k, k}$, and $\hat{H}_{R}^{k, k}$ coincides with the space of real-valued eigenfunctions with the $k$-th eigenvalue of the Laplacian on $\boldsymbol{P}_{n}(\mathrm{C})$.

First we shall prove Theorem 4.1 in the case of $S^{n}$. Let $\psi_{k}$ be the $k$-th standard minimal immersion of $S^{n}$. We fix the geodesic $\gamma$ of $S^{n}$. It is easily seen that $1+$ the order of $\psi_{k}$ is equal to the dimension of the minimum subspace of $\boldsymbol{R}^{m(k)+1}$ which contains the curve $\psi_{k}(\gamma)$. Let $\left\{f_{0} \circ \gamma, \cdots, f_{m(k)} \circ \gamma\right\}_{R}$ be the subspace of functions on $\gamma$ which is generated by the functions given by the restriction of the coordinate functions $f_{0}, \cdots, f_{m(k)}$ of $S^{n}$ on $\gamma$. Evidently the dimension of $\left\{f_{0} \circ \gamma, \cdots, f_{m(k)} \circ \gamma\right\}_{R}$ is equal to the dimension of the minimum subspace of $\boldsymbol{R}^{\boldsymbol{m}(k)+1}$ which contains $\psi_{k} \circ \gamma$. For simplicity, we take the geodesic $\gamma=S^{1}$ as follows: $S^{1}=$ $\left\{x \in S^{n} \subset \boldsymbol{R}^{n+1} ; x_{2}=x_{3}=\cdots=x_{n}=0\right\}$. Here we recall that the $k$-th eigenspace of the Laplacian on $S^{n}$ is given by the restriction of $H^{k}\left(R^{n+1}\right)$ on $S^{n}$ (Lemma 4.3). Putting $x_{2}=x_{3}=\cdots=x_{n}=0$, we get the linear homomorphism $\pi: H^{k}\left(\boldsymbol{R}^{n+1}\right) \rightarrow S^{k}\left(\boldsymbol{R}^{2}\right)$. It is easily checked that $\pi$ is surjective. It is wellknown that $\operatorname{dim} \boldsymbol{S}^{k}\left(\boldsymbol{R}^{2}\right)=k+1$ and hence $\operatorname{dim}\left\{f_{0} \circ \gamma, \cdots, f_{m(k)} \circ \gamma\right\}_{\boldsymbol{R}}=k+1$. Therefore the order of the $k$-th standard minimal immersion of $S^{n}$ into a unit sphere $S_{1}^{\boldsymbol{m}(k)}$ is $k$.

When $k_{1}, \cdots, k_{r}$ are all even or all odd, we assume that $k_{r}$ is the maximum of $k_{1}, \cdots, k_{r}$. We write the coordinate functions of $c_{j} \psi_{k_{j}}$ of $\psi_{k_{1}, \cdots, k_{r}}$ by $f_{0}^{j}, \cdots, f_{m\left(k_{j}\right)}^{j}$. By Lemmas 4.2 and $4.3,\left\{f_{0}^{j} \circ \gamma, \cdots, f_{m\left(k_{j}\right)}^{j} \circ \gamma\right\}_{R}$ is contained in $\left\{f_{0}^{r} \circ \gamma, \cdots, f_{m\left(k_{r}\right)}^{r} \circ \gamma\right\}_{R}$ for any $j=1, \cdots, r$. Thus we get

$$
\operatorname{dim}\left\{f_{0}^{1} \circ \gamma, \cdots, f_{m\left(k_{1}\right)}^{1} \circ \gamma, \cdots, f_{0}^{r} \circ \gamma, \cdots, f_{m\left(k_{r}\right)}^{r} \circ \gamma\right\}_{R} \leqq k_{r}+1 .
$$

If its dimension is less than $k_{r}+1, c_{r} \psi_{k_{r}}$, which is given by composing $\psi_{k_{1}, \cdots, k_{r}}$ and the projection $\boldsymbol{R}^{m\left(k_{1}\right)+\cdots+m\left(k_{r}\right)+r} \rightarrow \boldsymbol{R}^{m\left(k_{r}\right)+1}$ has the order less than $k_{r}$. It is a contradiction. Therefore the order of $\psi_{k_{1}, \cdots, k_{r}}$ is $k_{r}$. When $\left\{k_{1}, \cdots, k_{r}\right\}$ contains both even numbers and odd numbers, similarly we get $\operatorname{dim}\left\{f_{0}^{1} \circ \gamma, \cdots, f_{m\left(k_{r}\right)}^{r} \circ \gamma\right\}_{R}=$ the maximum of even numbers + the 
maximum of odd numbers +2 . Thus we obtain Theorem 4.1 in the case of $S^{n}$.

It is well-known that $\boldsymbol{P}_{n}(\boldsymbol{C})$ and $\boldsymbol{P}_{n}(\boldsymbol{H})$ contain an $n$-dimensional real projective space $\boldsymbol{P}_{n}(R)$ as a totally geodesic submanifold. We shall briefly recall it. Let $\boldsymbol{R}^{n+1}$ be included in $\boldsymbol{C}^{n+1}$ and $\boldsymbol{H}^{n+1}$ as a real part respectively. Let $\pi: S^{2 n+1} \subset C^{n+1} \rightarrow P_{n}(C)$ and $\pi: S^{4 n+8} \subset H^{n+1} \rightarrow P_{n}(H)$ be the Hopf fibrations. $S^{n}=R^{n+1} \cap S^{2 n+1}$ is a totally geodesic submanifold of $S^{2 n+1}$. Similarly $S^{n}=$ $\boldsymbol{R}^{n+1} \cap S^{4 n+3}$ is a totally geodesic submanifold of $S^{4 n+3}$. Restricting Hopf fibrations $\pi$ on $S^{n}$, we get totally geodesic immersions $\pi: S^{n} \rightarrow P_{n}(C)$ and $\pi: S^{n} \rightarrow P_{n}(\boldsymbol{H})$, and its image $\pi\left(S^{n}\right)$ is $P_{n}(R)$. Let $\psi_{k}$ be the $k$-th standard minimal immersion of $P_{n}(C)$ or $P_{n}(\boldsymbol{H}) . \quad \psi_{k}$ is a helical geodesic minimal immersion into a unit sphere. Trivially $\psi_{k} \circ \pi$ is also a helical geodesic immersion of $S^{n}$. Moreover the order of $\psi_{k}$ is equal to the order of $\psi_{k} \circ \pi$. So we shall calculate the order of the helical geodesic immersion $\psi_{k} \circ \pi$ of $S^{n}$. Restricting $H_{R}^{k, k}\left(C^{n+1}\right)$ on a real part $R^{n+1}$, we get a linear homomorphism $P: H_{R}^{k, k}\left(C^{n+1}\right) \rightarrow S^{2 k}\left(R^{n+1}\right)$. We remark that the homomorphism is not always surjective. But, for any $f \in H^{2 k}\left(\boldsymbol{R}^{n+1}\right)$, there exists $\tilde{f}$ in $H_{R}^{k, k}\left(C^{n+1}\right)$ such that $P \tilde{f}=f$. When $\psi_{k}$ is the $k$-th standard minimal immersion of $P_{n}(C)$, by the same arguments as $S^{n}$, we see that the order of $\psi_{k} \circ \pi$ is $2 k$, and hence the order of $\psi_{k}$ is $2 k$. In the case of $P_{n}(\boldsymbol{H})$, by the above argument we see that the order of the $k$-th standard minimal immersion $\psi_{k}$ is not greater than $2 k$. But it is known that the degree of $\psi_{k}$ is $2 k$ (Mashimo [7]). Since the order of $\psi_{k}$ is not less than the degree of $\psi_{k}$, we see that the order of $\psi_{k}$ is $2 k$. We can obtain the order of $\psi_{k_{1}, \cdots, k_{r}}$ by the same argument as the case of $S^{n}$.

\section{References}

[1] M. Berger, P. Gauduchon et E. Mazet, Le Spectre d'une Variété Riemannienne, Lecture Notes in Math., 194, Springer-Verlag, 1971.

[2] A. Besse, Manifolds All of Whose Geodesics are Closed, Ergebnisse der Math., 93, Springer, 1978.

[3] B. Y. Chen, Geometry of Submanifolds, Marcel Dekker Inc., New York, 1973.

[4] M. Do CARMO and N. WallaCh, Minimal immersions of spheres into spheres, Ann. of Math., 93 (1971), 43-62.

[5] J. ERBACHER, Reduction of the codimension of an isometric immersion, J. Differential Geometry, 5 (1971), 333-340.

[6] O. KowALSKI, Immersions of Riemannian manifolds with a given normal bundle structure I, Czechoslovak Math. J., 19 (94) (1969), 676-695.

[7] K. Mashimo, Degree of the standard isometric minimal immersions of the symmetric spaces of rank one into spheres, Tsukuba J. Math., 5 (1981), 291-297.

[8] H. NAKAGawa, On a certain minimal immersion of a Riemannian manifold into a sphere, Kodai Math. J., 3 (1980), 321-340. 
[9] K. Nomizu, Uniqueness of the normal connections and congruence of isometric immersions, Tôhoku Math. J., 28 (1976), 613-617.

[10] B. O'NeILl, Isotropic and Kaehler immersions, Canad. J. Math., 17 (1965), 907-915.

[11] K. SAKAмоTo, Planar geodesic immersions, Tôhoku Math. J., 29 (1977), 25-56.

[12] K. SaKamoto, Helical immersions into a unit sphere, Math. Ann., 261 (1982), 63-80.

[13] R. T. SMith, The spherical representations of groups transitive on $S^{n}$, Indiana Univ. Math. J., 24 (1974), 307-325.

[14] J. J. StoKER, Differential Geometry, Wiley Interscience, 1969.

[15] T. Takahashi, Minimal immersions of Riemannian manifolds, J. Math. Soc. Japan, 18 (1968), 203-215.

[16] K. TsuKADA, Isotropic minimal immersions of spheres into spheres, J. Math. Soc. Japan, 35 (1983), 355-379.

[17] N. Wallach, Minimal immersions of symmetric spaces into spheres, Symmetric Spaces, edited by W. M. Boothby and G. L. Weiss, New York, Marcel Dekker, 1972, 1-40.

Present Address:

DepartMent of Mathematics

Faculty of Sciences

Nigata UNIVERSITY

Nirgata 950-21 